\title{
The Case for Software-Defined Networking in Heterogeneous Networked Environments
}

\author{
Marc Mendonca \\ University of California \\ Santa Cruz, USA \\ msm@soe.ucsc.edu
}

\author{
Katia Obraczka \\ University of California \\ Santa Cruz, USA \\ katia@soe.ucsc.edu
}

\author{
Thierry Turletti \\ INRIA \\ Sophia Antipolis, France \\ Thierry.Turletti@inria.fr
}

\begin{abstract}
Motivated by a vision of a fully connected world, we explore how Software-Defined Networking (SDN) can be utilized to support heterogeneous environments consisting of both infrastructure--based and infrastructure-less networks. To make the case for SDN in heterogeneous networks, or Heterogeneous SDN (H-SDN), we examine application scenarios in which $\mathrm{H}-\mathrm{SDN}$ is a key enabling technology.

Categories and Subject Descriptors: C.2.1 [ComputerCommunications Networks]: Network Architecture and Design; C.2.3 [Computer-Communications Networks]: Network Operations
\end{abstract}

Keywords: Software-Defined Networks, SDN, Heterogeneous Networks, Wireless

\section{INTRODUCTION}

A critical enabling technology for future network services is support for a heterogeneous internet, which interconnects users and applications across networks ranging from wired, infrastructure-based wireless (e.g. cellular, wireless mesh), to infrastructure-less networks (e.g. MANET / VANET). Mobile traffic has been increasing exponentially over the past several years, with the number of mobile-connected devices soon to exceed the world's population. As such devices become commonplace, users will demand high-quality service regardless of location or access type.

A major challenge facing future networks is efficient utilization of resources; this is especially the case in MANETs as the available wireless capacity is inherently limited by a shared physical medium compounded by wireless channel impairments and the absence of a managed infrastructure. Though these self-organizing networks can be used in a heterogeneous environment to "fill the gaps" in an overburdened infrastructure, their lack of dedicated resources and shifting connectivity makes capacity sharing difficult. The heterogeneous characteristics of the underlying networks (e.g., physical medium, topology, stability) and nodes (e.g., buffer size,

Permission to make digital or hard copies of all or part of this work for personal or classroom use is granted without fee provided that copies are not made or distributed for profit or commercial advantage and that copies bear this notice and the full citation on the first page. To copy otherwise, to republish, to post on servers or to redistribute to lists, requires prior specific permission and/or a fee.

CoNEXT Student'12, December 10, 2012, Nice, France.

Copyright 2012 ACM 978-1-4503-1779-5/12/12 ...\$15.00. mobility) also add another important factor when considering routing and resource allocation.

Software-Defined Networking (SDN) has been proposed as a way to programmatically control networks by decoupling the control- from the data plane. This may allow rapid deployment of new applications and services aimed at decreasing costs or increasing security, stability, or availability in networks. OpenFlow[3] is a notable example of a SDN architecture based upon "programmable" switches which consist of: (1) a flow table containing entries along with an action to be invoked; and (2) a protocol for communication between the switch and a controller, a remote process that manages new flow table entries. SDN techniques to-date, such as OpenFlow, largely target infrastructure-based networks, especially those found in data centers. They promote a centralized control mechanism that is ill-suited to the level of decentralization and disruption in wireless environments.

Motivated by a vision of a fully connected world, in this paper, we explore how SDN can be utilized to support a hybrid environment consisting of both infrastructure-based and infrastructure-less networks. We call this new paradigm Heterogeneous-SDN, or H-SDN. While previous works [5, 1, 2 ] have examined the use of SDN in wireless environments, their scope has primarily focused on wireless infrastructure deployments (e.g., WiMAX, Wi-Fi) or mesh environments. However, to our knowledge no one has explored the challenges and benefits offered by extending the SDN paradigm in heterogeneous networked environments.

\section{USE CASES}

The heterogeneous environment examined in the following use cases consist of mobile devices of various capabilities but with limited- or intermittent connection to the network infrastructure, but are able to form ad hoc connections with other nearby devices. Additionally, some of the devices have multiple network interfaces (e.g., wired / 802.11 / cellular). In such environments, determining the effective network capacity is dependent on many factors beyond the physical medium; issues typically not encountered in planned infrastructure networks, such as diverse device characteristics (buffer size, mobility, impermanence, battery life) and network interoperability become important considerations.

We will examine two scenarios: a 'traditional' case and one whose network is SDN-enabled. In the SDN use case scenario, we will assume that the devices have agreed to some form of external control insofar as network decisions, such as buffering and routing, are concerned; although this raises 
several research challenges, we identify in each of the cases possible benefits to both users and infrastructure providers.

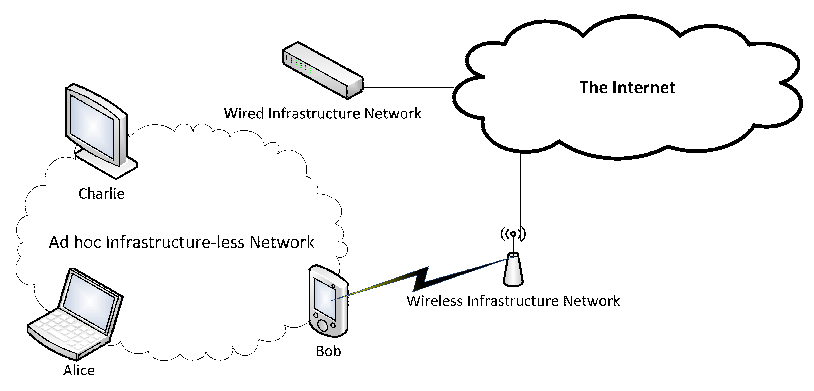

Figure 1: Sample topology

For the following use cases, we assume a topology similar to that of Figure 1. In the base case, a user "Alice" wishes to connect to the Internet and access the World Wide Web; unfortunately, she is unable to connect to infrastructure so she joins an ad hoc network instead. Another user "Bob" is connected to both the ad hoc and mobile data network.

Traditional: assuming the ad hoc network learns to route to Bob as a gateway, and Bob allows his device to be used as a NAT router, the service provider is unaware of Alice. Bob's connection is not assigned additional bandwidth, hindering performance; furthermore, Bob may be held responsible for Alice in case of data overages or illegal activity.

$S D N$ : The service provider is made aware when Alice joins the ad hoc network; therefore, it may decide to offer service to Alice via Bob and provision Bob's connection accordingly. The service provider may decide to sell Alice a temporary connection plan on the spot, or Alice may have an existing contract on another device; available resources, past user behavior, or any number of factors can be used in deciding whether to offer service to Alice. The service provider is thus able to maintain control of its network policy while being granted an opportunity for additional business. Alice is able to seamlessly connect to the Internet using a service plan. For his part, Bob may be offered incentives by the service provider, while avoiding performance loss or being held liable for Alice's traffic.

An extension to the base case is a scenario with multiple gateways. For example, shortly after Alice joins, a user "Charlie" with access to wired infrastructure also connects to the ad hoc network. In the traditional scenario, traffic is routed solely based on how the MANET protocol handles multiple gateways. In the SDN scenario, the network capacity can be managed based on the policies of the service providers and the characteristics of the available resources.

Next, we look at examples of service optimizations: In one possible situation, a group of users in the ad hoc network may be viewing the same content simultaneously (e.g., live streaming of a sport event). Using the base case from above, Bob is the link to the Internet from which the content originates. In the traditional scenario, optimizations such as caches or CDNs are performed either in the provider network or in the cloud; leaving Bob's link to the provider saturated with duplicate content. SDN enables routing policies to evolve and promotes the creation of new services; for example, it may be possible to reduce the strain on the overburdened infrastructure connection by caching content locally, or creating multicast streams on-the-fly for live content. Projects[4] have already started implement- ing information-centric networking over OpenFlow, demonstrating the feasability of tracing and delivering content using SDNs. Coupled with an awareness of the hybrid topology and available capacity of nodes within the network, popular content may be more efficiently stored and delivered to interested parties within the network.

Another possibility is greater support for mobile data offloading, in which other network interfaces are used for delivering packets originally destined for a cellular data network. Traditionally, this tends to be device initiated through periodic scanning for known complementary networks, such as a 802.11 access points previously visited by the user. With SDN, a provider may be able to remotely initiate offloading for heavy flows based on user proximity to complementary networks. Additionally, it may be able to seamlessly initiate the connection with a secured $\mathrm{Wi}-\mathrm{Fi}$ or Femtocell network that the user would have otherwise been unable to access.

Other possible use cases may evolve from emerging applications (e.g., participatory sensing, peer-to-peer gaming, vehicular communication) centered around local content or communication with nearby users.

\section{OPEN ISSUES AND CONCLUSION}

SDN has the potential to facilitate efficient deployment and management of network applications and services. To support the evolution of heterogeneous environments, SDN solutions must go beyond the current infrastructure-based SDN model and address several challenges, including deploying on end devices, handling multiple domains of control, supporting a flexible set of rules and actions, recognizing diverse device capabilities, tolerating delay and disruption, and integrating with other control planes. If properly implemented, the flexible management offered by SDN can help address problems that face hybrid networks, such as capacity sharing and inter-network compatibility.

The existence of independent forwarding devices and controllers raises other concerns, namely security, compatibility, and maintaining a balance of control and flexibility when devices cooperate to form a SDN. Policy issues, such as providing incentives for nodes to relinquish control or promoting cooperation between controllers, also need to be addressed.

The formulation of a $\mathrm{H}-\mathrm{SDN}$ framework to support heterogeneous networks would allow the coverage of existing infrastructure to expand while enabling innovation and interoperability in self-organizing networks, to the benefit of both users and providers.

\section{REFERENCES}

[1] A. Coyle and H. Nguyen. A frequency control algorithm for a mobile adhoc network. Military Communications and Information Systems Conference (MilCIS), November 2010.

[2] P. Dely, A. Kassler, and N. Bayer. Openflow for wireless mesh networks. IEEE International Conference on Computer Communications and Networks (ICCCN), 2011.

[3] N. McKeown, T. Anderson, H. Balakrishnan, G. Parulkar, L. Peterson, J. Rexford, S. Shenker, and J. Turner. Openflow: enabling innovation in campus networks. ACM SIGCOMM Computer Communication Review, 38(2):69-74, 2008.

[4] L. Veltri, G. Morabito, S. Salsano, N. Blefari-Melazzi, and A. Detti. Supporting information-centric functionality in software defined networks. IEEE ICC Workshop on Software Defined Networks, June 2012.

[5] K. Yap, R. Sherwood, M. Kobayashi, T. Huang, M. Chan, N. Handigol, N. McKeown, and G. Parulkar. Blueprint for introducing innovation into wireless mobile networks. ACM SIGCOMM Workshop on Virtualized Infrastructure Systems and Architectures, 2010. 